\title{
Influence of fs-laser desorption on target normal sheath accelerated ions
}

\author{
G. Hoffmeister, ${ }^{1, *}$ C. Bellei, ${ }^{2}$ K. Harres, ${ }^{1}$ D. Ivanov, ${ }^{3}$ D. Kraus, ${ }^{1}$ A. Pelka, ${ }^{1}$ B. Rethfeld,${ }^{3}$ G. Schaumann, ${ }^{1}$ and M. Roth ${ }^{1}$ \\ ${ }^{1}$ Institut für Kernphysik, Technische Universität Darmstadt, Schlossgartenstraße 9, 64289 Darmstadt, Germany \\ ${ }^{2}$ Lawrence Livermore National Laboratory, Livermore, California 94550, USA \\ ${ }^{3}$ Fachbereich Physik und Forschungszentrum OPTIMAS, Technische Universität Kaiserslautern, \\ Erwin-Schrödinger-Straße 46, 67663 Kaiserslautern, Germany
}

(Received 18 March 2013; published 24 April 2013)

\begin{abstract}
We report on the effects of fs-laser desorption on the ion acceleration induced by the target normal sheath acceleration (TNSA) mechanism. The experiment was performed at the Lawrence Livermore National Laboratory (LLNL) using the 100 TW Callisto laser of the Jupiter Laser Facility (JLF). Thin metal foils ( $\mathrm{Au}, \mathrm{Cu}$, and $\mathrm{Al}$ ) with thicknesses ranging from 10 to $20 \mu \mathrm{m}$ were irradiated by a variable number of low intensity $\left(\sim 10^{12} \mathrm{~W} / \mathrm{cm}^{2}\right)$ laser pulses, the last one arriving $100 \mathrm{~ms}$ before the main pulse. With these short pulses water vapor and hydrocarbon contaminations could stepwise be removed from the target surface. Substantial modifications of the TNSA-ion energy spectra were observed such as diminished proton energy and intensity, the absence of low-charged ion states, increased particle numbers for $\mathrm{C}^{4+}$ and $\mathrm{O}^{6+}$ ions in the higher energetic part of their particle spectra as well as the acceleration of target ions. The controlled application of fs-laser desorption on the laser-ion acceleration thus strongly influences the ion spectra and offers the possibility of selecting a targeted range of ion species for the acceleration to higher energies due to the systematic removal of contamination layers.
\end{abstract}

DOI: 10.1103/PhysRevSTAB.16.041304

PACS numbers: 52.38.Kd, 52.38.Mf

\section{INTRODUCTION}

The acceleration of ions generated by the interaction of ultraintense high power lasers (intensities of $I \geq$ $10^{19} \mathrm{~W} / \mathrm{cm}^{2}$ ) with matter has become a rapidly evolving field of interest. Many experiments in the past have demonstrated that laser-accelerated ion beams provide high quality characteristics such as high particle intensities $[1,2]$, short pulse lengths, and very low transverse source emittance [3] exceeding the parameters of standard particle accelerators. These qualities offer numerous potential applications reaching from ion sources of small dimensions over nuclear physics [4], high energy density physics like warm dense matter studies $[5,6]$ as well as its diagnostics [7] and inertial confinement fusion [8,9] to medical applications like laser-induced proton therapy $[10,11]$ in cancer treatment.

For future applications like the latter which aim for an ion beam quality comparable to conventional accelerators with respect to beam divergence and a monoenergetic particle spectrum, substantial improvements have to be developed. In order to meet these demands laseraccelerated ion beams have to be collimated and to be monoenergetic without losing substantially in brightness of the beam. These two requirements are nowadays the

\footnotetext{
*g.hoffmeister@gsi.de
}

Published by the American Physical Society under the terms of the Creative Commons Attribution 3.0 License. Further distribution of this work must maintain attribution to the author(s) and the published article's title, journal citation, and DOI. main challenges of experiments of ion acceleration by lasers and are the subject of intense research worldwide.

When laser pulses of relativistic intensities interact with a metal foil a plasma is instantaneously created by the laser's prepulse. The main pulse interacts with this plasma and accelerates the free electrons ponderomotively up to $\mathrm{MeV}$ energies in the direction of the laser propagation. Energetic electrons are able to penetrate the target foil and escape from the target rear side into vacuum, thus generating a quasistatic electric field on the order of a few $\mathrm{TV} / \mathrm{m}$. This electric field is strong enough to ionize the atoms on the target rear side and to accelerate them normal to the target surface. This mechanism is therefore named target normal sheath acceleration (TNSA) [12]. Without special target treatment and independently from the target material protons are always accelerated first as they have the highest charge-to-mass ratio. These protons stem from water vapor and hydrocarbon contaminations which are always present on the target surface due to the air contamination of the target. Protons from the topmost contamination layer on the target surface are exposed to the highest field gradients and screen the electric field for protons and ions coming from the successive layers. The acceleration of particles from different target depths results in a broad energy distribution which becomes broader with increasing contamination layer thickness. The inhomogeneous electron distribution in the sheath additionally leads to an inhomogeneous accelerating field in the transverse direction. The resulting exponential ion energy spectrum constitutes the main disadvantage in laser-ion acceleration. To date, there are only three groups that have produced a 
quasimonoenergetic ion beam with lasers and an energy spread of $20 \%$ or less [13-15].

Hegelich et al. [13] have used $20 \mu \mathrm{m}$ thick palladium foils that were resistively heated before the acceleration. At temperatures of about $600 \mathrm{~K}$, the targets were completely dehydrogenized, but carbon atoms were still remaining on the surface. By increasing the target's temperature $(T>1100 \mathrm{~K})$, the carbon underwent a phase transition and formed a monolayer graphite (graphene) on the Pd surface from which $\mathrm{C}^{5+}$ ions were accelerated to $3 \mathrm{MeV} / \mathrm{u}$ with an energy spread of $17 \%$. An advantage of resistive heating is the complete removal of all hydrogen at once but there are also several disadvantages: There is no possibility of studying the intermediate states between a full contamination layer on top of the target foil and a completely cleaned target surface, i.e., the formation of graphene could not be controlled. Furthermore, the setup requires a very precise temperature measurement.

The second group, Schwoerer et al. [14], has used $5 \mu \mathrm{m}$ thick titanium foils coated with $0.5 \mu \mathrm{m}$ thick hydrogenrich polymethyl methacrylate dots of $(20 \times 20) \mu \mathrm{m}^{2}$ on the target back surface. This configuration aimed on limiting the transversal extension of protons such that the proton-rich dot has a smaller diameter than the scale of inhomogeneity of the electron sheath. In this case, all protons experience the same potential. The parasitic proton contamination layer could be reduced by ns-laser ablation and the accelerated protons showed a quasimonoenergetic energy spectrum peaked at an energy of $1.2 \mathrm{MeV}$.

The third group, Ter-Avetisyan et al. [15], produced quasimonoenergetic deuteron bursts by the interaction of a high intensity, high contrast $\left(10^{-7}-10^{-8}\right)$ laser with limited mass water droplets. The peak position in the spectrum at $2 \mathrm{MeV}$ had an energy spread of 20\%. This experiment, however, suffered from the bad laser-droplet interaction probability.

In our experiment we concentrated on another more controllable method, laser-induced desorption, to clean the targets from their contaminations and to investigate its influence on the laser-accelerated ions. This method is a well-known technique in the field of mass spectroscopy developed by John B. Fenn and Koichi Tanaka to whom the chemistry Nobel prize 2002 [16] was awarded. Desorption from metallic surfaces with the help of femtosecond laser pulses is essentially triggered by excited electrons within the metallic substrate $[17,18]$. On femtosecond time scale, electrons obey a nonequilibrium energy distribution $[19,20]$. Higher occupied electron states in the substrate enable electron transfer to the adsorbate, thus inducing chemical reactions, i.e., desorption. On the femto- to picosecond time scale, also an enhanced electron temperature, as compared to the lattice, can induce desorption $[17,18]$. In contrast, for nanosecond irradiation heating proceeds slowly and electronic nonequilibrium plays no role. Here, thermal ablation of the contamination layer is likely to be the governing cleaning mechanism, which is, however, accompanied by thermal stresses and structural modifications of the metal. Molten material remains on the target surface, roughens it, and suppresses the TNSA [21]. Furthermore, the long pulse interacts with a plasma consisting of the ablated material. This leads to a loss in the ablation energy and cleaning efficiency [22]. These drawbacks are avoided in fs-laser desorption since heating of the lattice sets in when the laser pulse is already switched off.

The ultrashort time scales in the fs-laser desorption process enable one to remove monolayers of contaminants successively. In contrast to other target cleaning methods as resistive heating, also intermediate states between a fully contaminated target surface and a completely cleaned one are obtainable. This stepwise removal of adsorbates is essential for a better understanding of the TNSA. As the target surface structure strongly influences the generated charge states and maximum energies of the TNSA ions, information about the spatial decrease of the TNSA electric field potential into the target can be obtained. From the maximum ion energies the passed potential difference in the quasistatic electric field can be calculated. The location of the atoms in the target determines to what extent the accelerating field potential is experienced by them. Thus, it determines their degree of ionization and their subsequent acceleration. This understanding is indispensable for future laser-ion acceleration projects and will help a lot to controllably accelerate ions of distinct charge state in a certain energy window in the future.

\section{A. Target material composition and simulation}

For a first estimate of the required intensity of the desorption laser we have performed elastic recoil detection analysis (ERDA) investigations [23] to characterize the composition of the surface contamination of different metal foils. These measurements were carried out at GSI Helmholtzzentrum für Schwerionenforschung $\mathrm{GmbH}$ and at the University of the Germany Federal Armed Forces in Munich. During an ERDA run the target is hit by a heavy ion beam, in our case ${ }^{136} \mathrm{Xe}^{18+}$ with $1.4 \mathrm{MeV} / \mathrm{u}$, that ejects surface particles. These surface particles are analyzed by a magnetic spectrometer in case of $\mathrm{H}$ atoms and an ionization chamber in case of $\mathrm{C}$ and $\mathrm{O}$ atoms concerning their ion species, energy and yield. The ERDA measurements deliver information not only about the composition of the target contaminations but also about the particle concentration on the probe surface which is summed over the measurement depth of $\leq 1 \mu \mathrm{m}$. For the analyzed aluminum and copper target the obtained results are shown in Table I. The analysis of Pd, $\mathrm{Zr}$, and $\mathrm{W}$ target foils showed very similar numbers within the same order of magnitude [22]. Because of the higher oxidation potential of aluminum compared to copper we find a 2 times larger amount of oxygen atoms and only half the amount of carbon atoms 
TABLE I. Particle concentration $\rho_{\text {ERDA }}$ on the surface of aluminum and copper target foils of $20 \mu \mathrm{m}$ thickness summed over a measurement depth of $\leq 1 \mu \mathrm{m}$. The ERDA measurements were performed by a high resolution magnetic spectrometer for the analyzation of hydrogen and by an ionization chamber for the analyzation of carbon and oxygen.

\begin{tabular}{lcc}
\hline \hline Sample & Atom & $\rho_{\text {ERDA }}\left[\right.$ atoms $\left./ \mathrm{cm}^{2}\right]$ \\
\hline $\mathrm{Cu}$ & $\mathrm{H}$ & $1.91 \times 10^{16}$ \\
& $\mathrm{C}$ & $1.37 \times 10^{16}$ \\
& $\mathrm{O}$ & $1.61 \times 10^{16}$ \\
$\mathrm{Al}$ & $\mathrm{H}$ & $1.46 \times 10^{16}$ \\
& $\mathrm{C}$ & $6.94 \times 10^{15}$ \\
& $\mathrm{O}$ & $3.03 \times 10^{16}$ \\
\hline \hline
\end{tabular}

per square centimeter on the aluminum foil surface compared to the copper foil surface. The hydrogen concentration of both aluminum and copper surfaces is nearly equal.

We calculate the appropriate laser fluences to clean the surface from contaminations with the help of a numerical approach [24], combining the two-temperature model (TTM) and the molecular dynamics (MD) method. While the TTM accounts for an accurate description of the initially heated electronic system and its energy transfer to the lattice $[25,26]$, the MD method focusses on the atomic dynamics like melting and ablation. Here, we aim to avoid damage of the metallic substrate and therefore calculate the absorbed laser fluences $F_{\text {abs }}$, chosen to be $10 \%$ below the melting threshold. The corresponding intensities $I_{\text {abs }}$ are calculated for the 90 fs Callisto laser beam. These values refer to the absorbed laser fluences $F_{\text {abs }}=F_{\text {in }}(1-R)$, not to the incoming laser fluences $F_{\text {in }}$, where $R$ denotes the reflectance. The reflection of laser light on the target surface is not taken directly into account in our simulation. Instead, values of the reflectance varying from $R=0.87$ (aluminum) to $R=0.97$ (copper and gold), corresponding to pure metallic surfaces are assumed. The calculated fluences and laser intensities at $10 \%$ below melting threshold are given in Table II.

After the cleaning by the desorption laser the readsorption process starts. For a constant temperature the readsorption on the target surface is governed by the pressure in the target chamber [27] and the time delay between the desorption laser and the high energy laser beam driving the

TABLE II. Required absorbed laser fluences $F_{\text {abs }}$ for cleaning the surfaces of the different materials. The values are chosen to be $10 \%$ below the melting threshold. The absorbed intensities $I_{\mathrm{abs}}$ and the corresponding incoming intensities $I_{\text {in }}$ are calculated for the 90 fs Callisto laser beam assuming reflectance values of $R=0.87$ for aluminum and $R=0.97$ for copper and gold.

\begin{tabular}{lccc}
\hline \hline Sample & $F_{\text {abs }}\left[\mathrm{J} / \mathrm{cm}^{2}\right]$ & $I_{\text {abs }}\left[\mathrm{W} / \mathrm{cm}^{2}\right]$ & $I_{\text {in }}\left[\mathrm{W} / \mathrm{cm}^{2}\right]$ \\
\hline $\mathrm{Au}$ & 0.0765 & $8.50 \times 10^{11}$ & $2.83 \times 10^{13}$ \\
$\mathrm{Cu}$ & 0.0250 & $2.78 \times 10^{11}$ & $9.26 \times 10^{12}$ \\
$\mathrm{Al}$ & 0.0120 & $1.33 \times 10^{11}$ & $1.03 \times 10^{12}$ \\
\hline \hline
\end{tabular}

ion acceleration. According to the kinetic gas theory the flux of residual gas particles that hit the target surface is given by

$$
F=\frac{N_{A} p}{\sqrt{2 \pi M R_{m} T}} \simeq 2.8 \times 10^{20} \frac{p[\mathrm{mbar}]}{\mathrm{cm}^{2} \cdot \mathrm{s}},
$$

where $p$ is the pressure in the target chamber in units of mbar, $M$ the molecular mass of the gas, assumed here to be $30.3 \mathrm{~g} / \mathrm{mol}$ according to the normal air composition, $R_{m}$ the molar gas constant, $T$ the temperature in $K$, estimated here to $298 \mathrm{~K}$, and $N_{A}$ the Avogadro constant, respectively. Metallic surfaces contain on the order of magnitude of $10^{15}$ atoms $/ \mathrm{cm}^{2}$. Assuming a pressure of $3.6 \times 10^{-6}$ mbar in the chamber a surface atom experiences one collision per second. With an adhesion coefficient of one it takes about one second at this pressure until the target surface is covered with a monolayer of adsorbates. However, the adhesion coefficient gets smaller the more surface atoms are covered.

\section{EXPERIMENTAL SETUP}

The experiment was performed at the Jupiter Laser Facility (JLF) of the Lawrence Livermore National Laboratory (LLNL) using the $100 \mathrm{TW}$ Callisto laser. This ultraintense high power Ti:sapphire laser has a laser pulse duration of about $90 \mathrm{fs}$ at a wavelength of $800 \mathrm{~nm}$. The chirped pulse amplification (CPA) beam provides pulse energies up to $11 \mathrm{~J}$ at an intensity contrast of the $1 \mathrm{~ns}$ amplified spontaneous emission pedestal to the main pulse of $10^{-7}-10^{-8}$. It was focused on the target by a $f / 3$ off axis parabola at $34^{\circ}$ incidence angle. The focal spot diameter was $15 \mu \mathrm{m}$ which corresponds to a maximum laser intensity of $6.9 \times 10^{19} \mathrm{~W} / \mathrm{cm}^{2}$.

For the modification of the target surface by a successive removal of contaminants laser desorption pulses of energies between 1-2 mJ irradiated thin metal foils of gold, copper (both $10 \mu \mathrm{m}$ ) and aluminum (11 or $20 \mu \mathrm{m}$ ) shortly before the CPA beam initiated the TNSA process. These desorption pulses were delivered from the same front end as the CPA beam with a repetition rate of $10 \mathrm{~Hz}$. Throughout the experiment the following parameters of the laser desorption pulses were varied: The focal spot size of the desorption beam was $450 \mu \mathrm{m}$ or $800 \mu \mathrm{m}$, leading to intensities of $I_{\mathrm{des}} \approx 10^{12}-10^{13} \mathrm{~W} / \mathrm{cm}^{2}$, and the number of applied desorption pulses changed. For some shots, the desorption beam was split into two beamlets directed on the target front and rear side. The additional front side irradiation was applied in order to suppress proton acceleration from the target front side. The CPA beam arrived at the mostly cleaned target with a time delay of $100 \mathrm{~ms}$ after the last desorption pulse and triggered the TNSA mechanism.

The measurement and the analysis of the accelerated ions were done by a Thomson parabola spectrometer [28] under $0^{\circ}$ from target normal with a $(90 \times 70) \mathrm{mm}^{2}$ microchannel plate (MCP) for an online measurement. 
Radiochromic films in stack configuration [29] were used as a second independent diagnostics for the registration of the accelerated protons. The Thomson parabola constituted the main diagnostics for analyzing the individual ion signals. Its constant electric and magnetic fields deflect the incoming ions according to their kinetic energy and their charge-to-mass ratio on their way to the MCP where their signal becomes amplified, gets registered on a phosphor screen and is recorded by a CCD camera. In the Thomson parabola images the intensity of each curve represents the energy dependent particle current with highest particle energies near the origin due to smallest deflections by the applied electromagnetic fields.

\section{RESULTS}

In the following, we present the observed influences of fs-laser desorption on the ion acceleration for the three investigated target foil materials $\mathrm{Au}, \mathrm{Cu}$ and $\mathrm{Al}$. Systematic studies were performed for $\mathrm{Au}$ foils which are discussed in the first subsection. Experiments on $\mathrm{Cu}$ and $\mathrm{Al}$ foils confirm those results, as shown subsequently.

\section{A. Au foils}

Figure 1(a) shows a reference shot, i.e., the CPA beam hit the target and induced the TNSA without any prior laser desorption. Apart from protons leaving the most intense particle trace on the MCP, $\mathrm{C}^{4+}$ was favorably produced and accelerated. This is due to the higher charge-to-mass ratio than $\mathrm{C}^{+}, \mathrm{C}^{2+}$, and $\mathrm{C}^{3+}$ but lower ionization potential than $\mathrm{C}^{5+}$. In addition to carbon ions we also observed traces of oxygen ions with lower particle intensities. Gold ions could not be accelerated indicating that the contamination layer was too thick. The contaminantshere in the form of protons, carbon, and oxygen



(a) shot 1
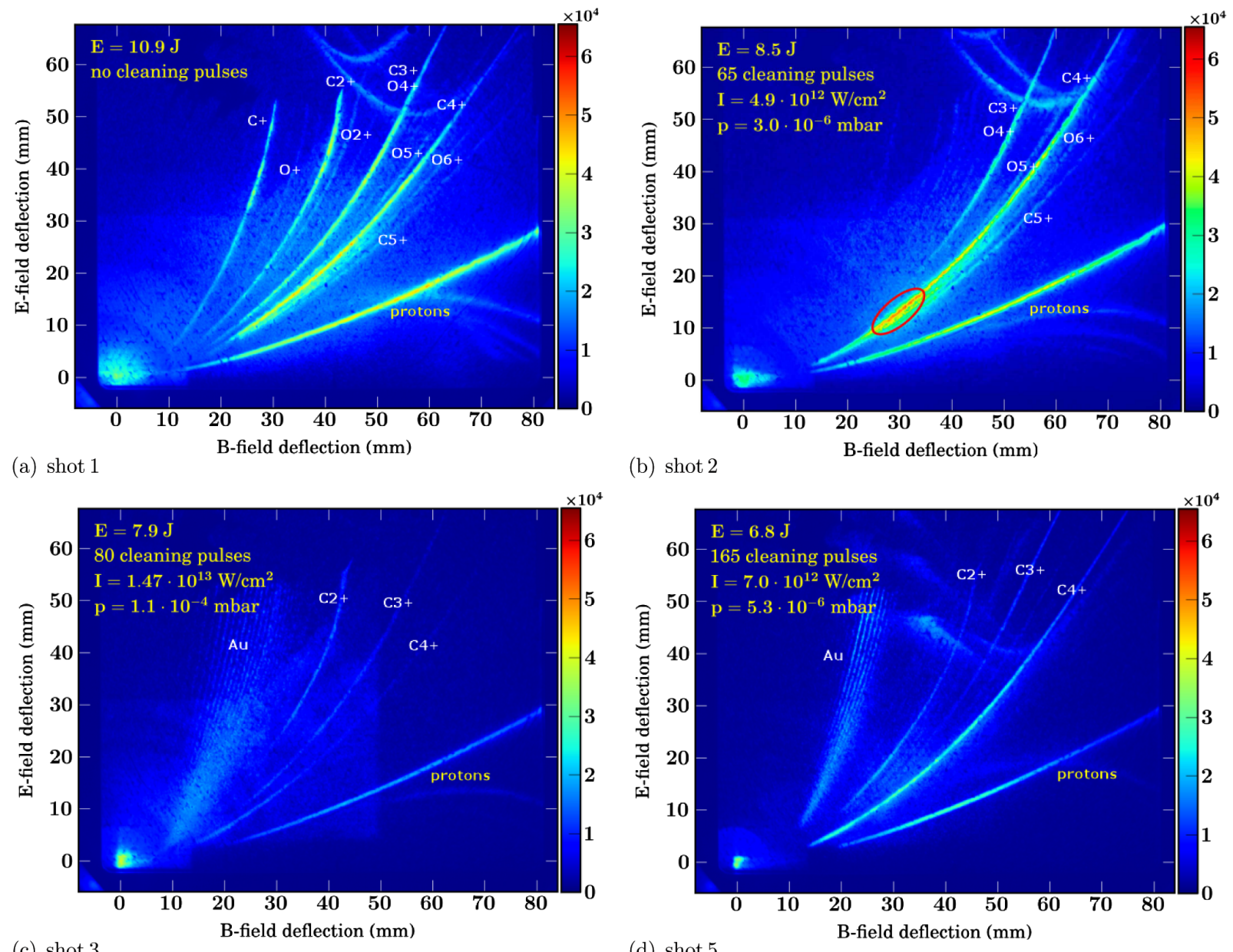

(b) $\operatorname{shot} 2$

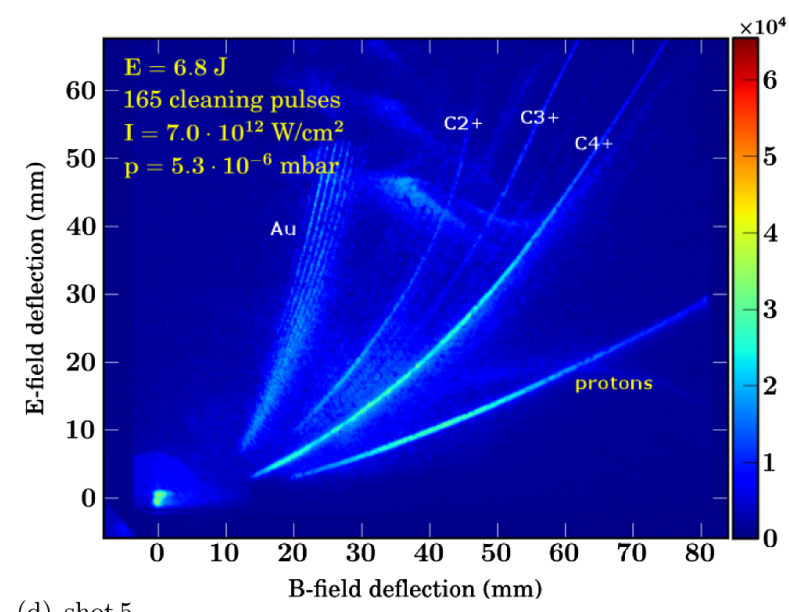

(d) $\operatorname{shot} 5$

FIG. 1. Thomson parabola images of shots on $10 \mu \mathrm{m}$ thick Au foils with different cleaning pulse configurations and target chamber pressures. The signal intensity scale in MCP counts is given on the right. In (a), a reference shot without any cleaning pulses is shown. Besides an intense proton signal carbon ions up to charge state 5+ and oxygen ions are present. In (b), at a desorption beam intensity of $I_{\text {des }}=4.9 \times 10^{12} \mathrm{~W} / \mathrm{cm}^{2}$, ions with low charge states vanish completely while $\mathrm{C}^{4+}$ has a higher maximum energy and shows an enhanced signal between 0.18 and $0.35 \mathrm{MeV} / \mathrm{u}$ marked by the red ellipse. Increasing the intensity to $I_{\text {des }}=1.47 \times 10^{13} \mathrm{~W} / \mathrm{cm}^{2}$, like in (c), or the number of cleaning pulses to 165 at $I_{\text {des }}=7.0 \times 10^{12} \mathrm{~W} / \mathrm{cm}^{2}$, like in (d), the proton signal and their maximum energy clearly decrease compared to (a) and gold ions are accelerated. 
ions-screen the TNSA field from the target atoms and prevent the latter from getting field ionized and from getting accelerated. The maximum proton and carbon ion energies for this reference shot, labeled in the following as shot 1, are listed in Table III.

The application of laser desorption changes the TNSAion spectra strongly. This is shown in the Thomson parabola images, Figs. 1(b)-1(d), for different laser shot parameters. The respective shot parameters and their influence on the ion acceleration are given in detail in Table III. Figure 2 displays the obtained ion spectra of $\mathrm{C}^{3+}$ and $\mathrm{C}^{4+}$ corresponding to the listed shots. The proton spectra are shown in Fig. 3 which includes also a shot on a copper foil and on an aluminum foil.

Like shot 2 in Fig. 1(b) shows, at a moderate number of cleaning pulses with an intensity $I_{\text {des }} \simeq 4.9 \times 10^{12} \mathrm{~W} / \mathrm{cm}^{2}$ of the desorption laser the proton acceleration was hardly affected by the laser desorption. In contrast, the particle spectra of carbon and oxygen ions got strongly modified: Compared to shot 1 , carbon and oxygen ions with low charge states $\left(\mathrm{C}^{+}, \mathrm{C}^{2+}, \mathrm{O}^{+}, \mathrm{O}^{2+}, \mathrm{O}^{3+}\right)$ completely disappeared and the maximum energy as well as the total amount of the accelerated $\mathrm{C}^{3+}$ ions decreased substantially. But the $\mathrm{C}^{4+}$ ions gained a maximum kinetic energy which was twice as much as in shot 1 . An enhanced signal intensity in the $\mathrm{C}^{4+}$-ion trace was noticed for particle energies between 0.18 and $0.38 \mathrm{MeV} / \mathrm{u}$, indicated by the red ellipse in Fig. 1(b).

These features change even more for an increased desorption process with an intensity $I_{\text {des }} \geq 7.0 \times$ $10^{12} \mathrm{~W} / \mathrm{cm}^{2}$ of the desorption laser and with more cleaning pulses. Like both the Thomson parabola images of shot 3 and shot 5, Figs. 1(c) and 1(d), and the proton spectra in Fig. 3 demonstrate, the proton signal intensity on the MCP decreased by about one order of magnitude compared to shot 1 . A substantial loss in the proton maximum energy by at least a factor of 2 was noted, too. Less but higher energetic cleaning pulses like in shot 3 seem to be more effective for getting rid of the hydrogen contamination than more cleaning pulses with lower pulse energy as in shot 5 .

The influence of the enhanced desorption process on the carbon ions differs for their individual charge states. Like Fig. 2 shows, for $\mathrm{C}^{3+}$ a similar behavior as for the protons is recorded. Both the particle intensity and the maximum energy of $\mathrm{C}^{3+}$ drop compared to shot 1 .

Exactly the opposite is observed for $\mathrm{C}^{4+}$. The maximum energy of $\mathrm{C}^{4+}$ is shifted to higher energies and an increased particle intensity in the higher energetic region is noted in comparison to shot 1. Like Fig. 2 illustrates, the total particle intensity of $\mathrm{C}^{4+}$, however, was strongly affected by the respective cleaning performance. With increasing

TABLE III. Overview of all important parameters of the discussed laser desorption shots and of the reference shot (no cleaning) on $10 \mu \mathrm{m}$ Au foils. The quantities listed here are the intensity $I_{\mathrm{des}}$ and the energy $E_{\mathrm{des}}$ of the cleaning pulses, the laser energy $E_{\mathrm{CPA}}$ of the main pulse and the target chamber pressure $p$. Included are also the analyzed results from the Thomson parabola ion traces regarding the maximum ion kinetic energies $E_{i}^{\max }$ and a calculated estimate for the maximum potential difference $\Delta \Phi_{\text {ion }}=E_{i}^{\max } / Z e$ the fastest ions should have experienced by the quasistatic electric field.

\begin{tabular}{|c|c|c|c|c|c|c|c|c|}
\hline Shot no. & \# pulses & $I_{\mathrm{des}}\left[\mathrm{W} / \mathrm{cm}^{2}\right]$ & $E_{\mathrm{des}}[\mathrm{mJ}]$ & $E_{\mathrm{CPA}}[\mathrm{J}]$ & $p$ [mbar] & Ions & $E_{\mathrm{kin}}^{\max }[\mathrm{MeV} / \mathrm{u}]$ & $\Delta \Phi_{\text {ion }}[\mathrm{MV}]$ \\
\hline \multirow[t]{6}{*}{1} & 0 & & & 10.9 & & $p$ & $12.00 \pm 1.00$ & $12.00 \pm 1.00$ \\
\hline & & & & & & $\mathrm{C}^{5+}$ & $0.38 \pm 0.04$ & $0.90 \pm 0.10$ \\
\hline & & & & & & $\mathrm{C}^{4+}$ & $0.58 \pm 0.08$ & $1.75 \pm 0.25$ \\
\hline & & & & & & $\mathrm{C}^{3+}$ & $0.35 \pm 0.04$ & $1.40 \pm 0.17$ \\
\hline & & & & & & $\mathrm{C}^{2+}$ & $0.21 \pm 0.02$ & $1.25 \pm 0.10$ \\
\hline & & & & & & $\mathrm{C}^{+}$ & $0.13 \pm 0.01$ & $1.50 \pm 0.10$ \\
\hline \multirow[t]{4}{*}{2} & 65 (rear side) & $4.9 \times 10^{12}$ & 2.1 & 8.5 & $3 \times 10^{-6}$ & $p$ & $12.00 \pm 1.00$ & $12.00 \pm 1.00$ \\
\hline & & & & & & $\mathrm{C}^{5+}$ & $0.45 \pm 0.02$ & $1.10 \pm 0.04$ \\
\hline & & & & & & $\mathrm{C}^{4+}$ & $1.17 \pm 0.08$ & $3.50 \pm 0.25$ \\
\hline & & & & & & $\mathrm{C}^{3+}$ & $0.15 \pm 0.03$ & $0.60 \pm 0.10$ \\
\hline \multirow[t]{4}{*}{3} & 80 (rear side) & $1.47 \times 10^{13}$ & 2.3 & 7.9 & $1.1 \times 10^{-4}$ & $p$ & $3.40 \pm 0.40$ & $3.40 \pm 0.40$ \\
\hline & & & & & & $\mathrm{C}^{4+}$ & $0.96 \pm 0.08$ & $2.88 \pm 0.25$ \\
\hline & & & & & & $\mathrm{C}^{3+}$ & $0.18 \pm 0.03$ & $0.70 \pm 0.10$ \\
\hline & & & & & & $\mathrm{C}^{2+}$ & $0.06 \pm 0.01$ & $0.35 \pm 0.06$ \\
\hline \multirow[t]{3}{*}{4} & 100 (rear and front side) & $7.0 \times 10^{12}$ & 1 & 5.6 & $3.1 \times 10^{-5}$ & $p$ & $3.20 \pm 0.20$ & $3.20 \pm 0.20$ \\
\hline & & & & & & $\mathrm{C}^{4+}$ & $0.67 \pm 0.08$ & $2.00 \pm 0.25$ \\
\hline & & & & & & $\mathrm{C}^{3+}$ & $0.12 \pm 0.02$ & $0.47 \pm 0.07$ \\
\hline \multirow[t]{4}{*}{5} & 165 (rear and front side) & $7.0 \times 10^{12}$ & 1 & 6.8 & $5.3 \times 10^{-6}$ & $p$ & $4.50 \pm 0.50$ & $4.50 \pm 0.50$ \\
\hline & & & & & & $\mathrm{C}^{4+}$ & $1.10 \pm 0.03$ & $3.30 \pm 0.10$ \\
\hline & & & & & & $\mathrm{C}^{3+}$ & $0.17 \pm 0.01$ & $0.68 \pm 0.05$ \\
\hline & & & & & & $\mathrm{C}^{2+}$ & $0.13 \pm 0.01$ & $0.80 \pm 0.05$ \\
\hline
\end{tabular}




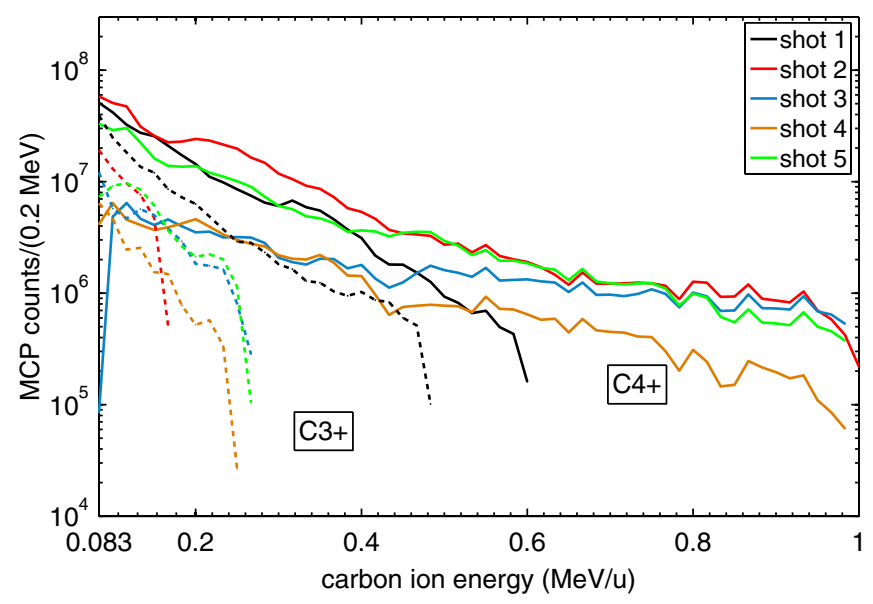

FIG. 2. Comparison of all $\mathrm{C}^{4+}$ (solid curves) and $\mathrm{C}^{3+}$ (dashed curves) spectra obtained from the discussed shots on Au foils, see Table III. While the maximum energy of $\mathrm{C}^{4+}$ increases when laser desorption is applied, it decreases in the case of $\mathrm{C}^{3+}$ compared to the reference shot (shot 1).

intensity of the desorption laser beam (shot 3) and/or low CPA pulse energy (shot 4) the $\mathrm{C}^{4+}$-ion flux diminished, especially for energies below $0.5 \mathrm{MeV} / \mathrm{u}$. The opposite happened for lower intensities of the desorption laser beam and/or higher CPA pulse energy (shot 5 and shot 2) showing an increased particle intensity in that region.

The parabola traces of the carbon ions with low charge states, i.e. $\mathrm{C}^{+}$and $\mathrm{C}^{2+}$, vanished almost completely on the $\mathrm{MCP}$ due to the laser desorption.

For each shot listed in Table III, Fig. 4 illustrates the fraction of the individual carbon ion charge states to the total carbon ion yield, integrated in energy. The fraction of accelerated $\mathrm{C}^{4+}$ ions relatively increases due to the laser desorption while the relative amount of the carbon ions with charge states lower than $4+$ decreases or even is



FIG. 3. Comparison of all proton spectra discussed in the text. The shot details for gold foils are given in Table III. A definite decrease in proton signal intensity was noted for shots with preceding desorption pulses of $I_{\mathrm{des}} \geq 7.0 \times 10^{12} \mathrm{~W} / \mathrm{cm}^{2}$.

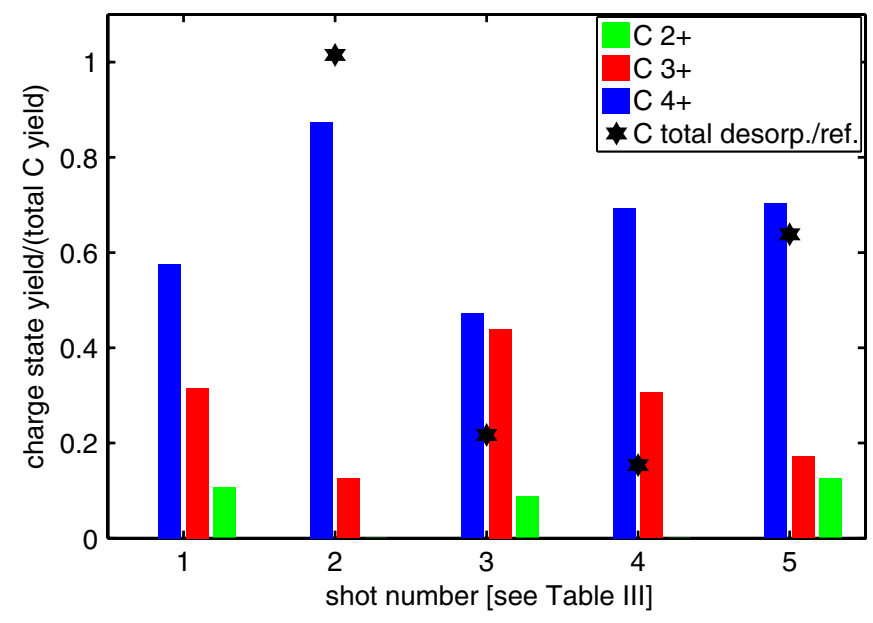

FIG. 4. Fraction of the $\mathrm{C}^{4+}, \mathrm{C}^{3+}$, and $\mathrm{C}^{2+}$ ion yields to the total carbon ion yield of each shot, integrated from 0.083 to $1 \mathrm{MeV} / \mathrm{u}$. The black stars show the total carbon ion yield ratio of the individual shots with desorption to the reference shot (shot 1).

zero. This feature was most distinctive in shot 2 with an intermediate intensity of the desorption laser. The slight deviation of shot 3 from this trend is probably explainable by the very low general carbon ion signal in this shot. The ratio of the total carbon ion yield, i.e., summed over all charge states, of the shots with applied laser desorption to the reference shot is indicated by the black stars in Fig. 4. They mark the general reduction of accelerated carbon ions in the shots of preceded laser desorption with $I_{\text {des }} \geq 7.0 \times$ $10^{12} \mathrm{~W} / \mathrm{cm}^{2}$ (shots $3,4,5$ ), indicating that the carbon density on the target surface was diminished by the desorption.

Apart from the discussed modifications in the proton and carbon ion spectra, the intensified cleaning process led also to the acceleration of gold ions. Their charge states are ranging from $\mathrm{Au}^{3+}$ to $\mathrm{Au}^{20+}$ in shot 3 [Fig. 1(c)] and from $\mathrm{Au}^{8+}$ to $\mathrm{Au}^{15+}$ in shot 5 [Fig. 1(d)].

\section{B. $\mathrm{Cu}$ foils}

Laser desorption of copper foil targets confirms the results obtained from gold foils. As the Thomson parabola image, Fig. 5(a), demonstrates, the irradiation of both target surfaces with 100 cleaning pulses of $I_{\text {des }}=7.0 \times$ $10^{12} \mathrm{~W} / \mathrm{cm}^{2}$ led to the complete absence of carbon and oxygen ions with low charge states and to reduced signals of those with higher charge states. Even the intense signals of $\mathrm{C}^{4+}$ ions disappeared. Like already observed for shots on gold foils, the maximum energy of protons decreased to half of its value and their intensity was reduced by one order of magnitude compared to shot 1 (see Fig. 3). An abundant spectrum of copper lines was evident on the Thomson parabola which was not present in shots without laser desorption. The various $\mathrm{Cu}$-ion traces ranged from $\mathrm{Cu}^{2+}$ up to $\mathrm{Cu}^{14+}$. On a closer look, the two copper 

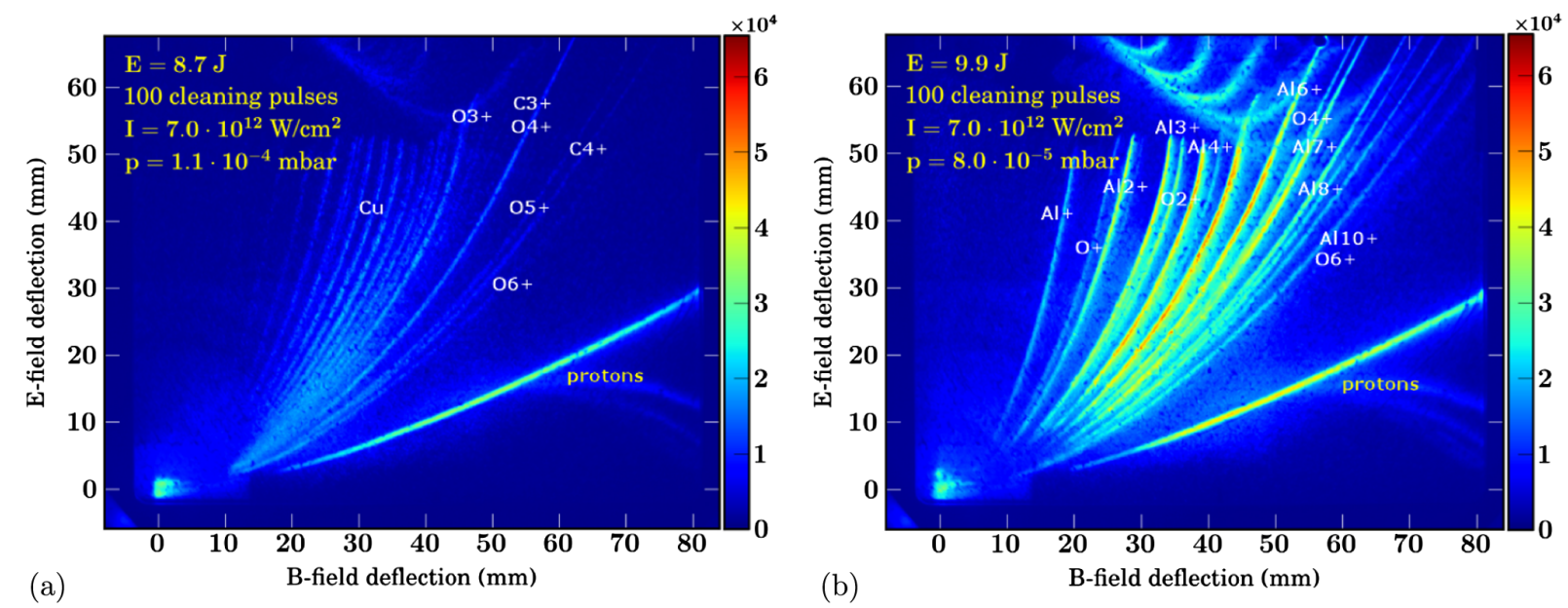

FIG. 5. Thomson parabola images of a shot on a $\mathrm{Cu}$ foil with a CPA pulse energy of $8.7 \mathrm{~J}$ in (a), and on an Al foil with a CPA pulse energy of $9.9 \mathrm{~J}$ in (b) are shown. In both cases, 100 cleaning pulses on both target surfaces were irradiating the target prior to the CPA pulse.

isotopes ${ }^{63} \mathrm{Cu}$ and ${ }^{65} \mathrm{Cu}$ could even be discerned. These results show that several monolayers of contaminants have been removed from the target surface and the TNSA field was sufficiently high to accelerate copper ions.

\section{Al foils}

Very dominant effects of the fs-laser desorption were observed when using $11 \mu \mathrm{m}$ thin aluminum targets. The Thomson parabola image, Fig. 5(b), shows that numerous intense oxygen and aluminum ion traces of various charge states were recorded. The high oxidation potential of aluminum in comparison to gold or copper makes oxygen the predominant surface contamination. Twice as much oxygen as hydrogen and 4 times as much oxygen as carbon



FIG. 6. Comparison of the accelerated oxygen and aluminum ions obtained from a shot on a $11 \mu \mathrm{m}$ thick $\mathrm{Al}$ foil. The ion traces of $\mathrm{O}^{2+}, \mathrm{Al}^{3+}$, and $\mathrm{Al}^{4+}$ could be analyzed individually while the other ion traces could only be analyzed as a summed particle signal due to superpositions. $\mathrm{O}^{6+}$ superposed with $\mathrm{Al}^{10+}$ were the ions exposed to the highest electric field potential with an acceleration of up to $1.94 \mathrm{MeV} / \mathrm{u}$. atoms per square centimeter resulted from the ERDA run (see Sec. IA) of the Al surface investigation and could qualitatively be confirmed. Besides the oxygen ions, abundant aluminum ion traces were visible which reach from $\mathrm{Al}^{+}$to $\mathrm{Al}^{10+}$ while the proton signal shows the common feature of loss in both intensity and maximum energy (Fig. 3). Figure 6 demonstrates the particle energy spectra of almost all oxygen ions and most of the aluminum ions illustrating that they are continuously accelerated to higher maximum energies with increasing charge states. The electron binding energies of oxygen in the $K$ shell are more than 10 times higher than in the $L$ shell. Therefore, $\mathrm{O}^{7+}$ and $\mathrm{O}^{8+}$ could not be accelerated with the applied CPA pulse energies. The same argument is valid for aluminum ions. Higher charge states than $\mathrm{Al}^{11+}$ leaving a too weak trace to be analyzed on the phosphor screen were also not available for the TNSA. Because of a very similar charge-to-mass ratio we got superpositions of aluminum ion traces of charge state $\mathrm{Al}^{5+}$ and higher with oxygen ion traces of charge state $\mathrm{O}^{3+}$ and higher. This renders a careful analysis of their single particle intensities difficult and led to overestimated particle signals. Those ion traces affected by superpositions remain nevertheless comparable to each other and the maximum energy of the ions with a fixed charge-to-mass ratio could be determined as well. They fit in the common picture of the laser desorption results that the acceleration of ions from the target material considerably benefits from the reduced hydrogen content on the target surface.

\section{Discussion}

The surface structure on the target rear side which acts as the ion source determines the characteristic of the TNSA. Ions with highest charge-to-mass ratio and with an exceedable ionization potential are preferably generated and accelerated. At the beginning of the acceleration process, 
the TNSA electric field energy is therefore transferred into the acceleration of protons, followed by $\mathrm{C}^{5+}$ and $\mathrm{C}^{4+}$ according to their density distribution on the target rear side. The electric field becomes screened for atoms which are located deeper in the target foil. As illustrated in Fig. 7, left sketch, these atoms experience a lower electric field which, however, is strong enough to further ionize them to lower charge states and to accelerate these ions. In case of the reference shot we therefore observe the acceleration of $\mathrm{C}^{+}, \mathrm{C}^{2+}, \mathrm{O}^{+}, \mathrm{O}^{2+}$, and $\mathrm{O}^{3+}$ but not of gold, copper, or aluminum ions which experience a too low TNSA electric field.

The cleaning pulses modify the target surface structure by successively removing the adsorbed contaminants. Higher intensities of the desorption laser pulses seem to be more effective than a higher number of pulses like the comparison of shot 3 to shot 5 on gold foils illustrates. Apart from the readsorption of contaminants between the individual laser pulses, a net heat transfer to the target surface sets in as more and more cleaning pulses continue to irradiate the target. The efficiency of desorbing contaminants drops and a saturation effect sets in between 150 and 200 cleaning pulses. Although these two circumstances decelerate the desorption process, the net effect results in a stepwise decrease of the contamination layer thickness when remaining below that saturation limit. Like sketched in the right image of Fig. 7, the thinner contamination layer leads to less screening effects of the TNSA electric field by contaminants. Because of the reduced proton density on the target rear side, a larger accelerating field is available for ionizing carbon and oxygen atoms to higher charge states and for accelerating them to higher energies. This was the case for $\mathrm{C}^{4+}$ in our presented shot results on gold foils with preceding laser desorption. At a moderate intensity of the desorption laser beam and an intermediate number of pulses the contamination layer decreases modestly and screening effects still play a major role. Like it
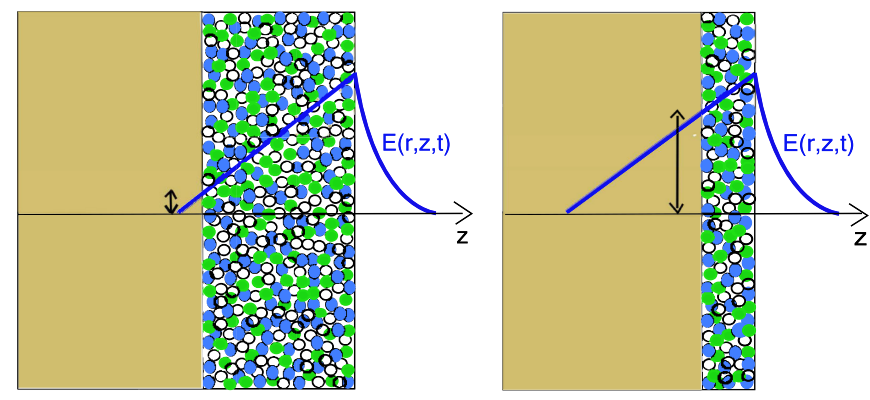

FIG. 7. C-H-O contamination layer on the target surface (not true to scale) and the electric field $E(r, z, t)$ reaching into the foil: no laser desorption (left) and with laser desorption (right). Because of the thinner contamination layer in the right image, metal atoms experience a sufficiently strong electric field to get ionized and accelerated. This is not the case in the left image, where the electric field gets strongly screened by the contaminants. was observed in shot 2, Fig. 1(b), the remaining field potential did not suffice to ionize gold atoms but an enhanced acceleration of $\mathrm{C}^{4+}$ occurred. The observed reduction or even absence of the carbon and oxygen ions with low charge states results from the fact that the residual field potential still suffices for the ionization of carbon to higher charge states, in our case $\mathrm{C}^{4+}$, and for their acceleration to higher energies. With increasing intensity of the desorption laser beam the contamination layer gets thinner and thinner and screening effects by contaminants become less important for the TNSA of metal ions. When there is no carbon and oxygen left anymore on the target surface and the remaining field potential is still sufficiently high it ionizes gold and copper atoms and accelerates these ions. Their maximum charge state is determined by the strength of the remaining electric field potential. We observe those gold ions in shot 3 and shot 5, Figs. 1(c) and 1(d), and the respective copper ions in Fig. 5(a). In case of aluminum foils, we find both strong aluminum and oxygen ions which is due to the thicker oxide layer on aluminum than on copper or gold foils.

In our presented shot on aluminum the superposed ion species $\mathrm{O}^{6+}$ and $\mathrm{Al}^{10+}$ experienced an acceleration up to $E_{\mathrm{kin}}^{\max }=(1.94 \pm 0.12) \mathrm{MeV} / \mathrm{u}$. Such maximum kinetic energy can be reached by these ions, assuming a passed potential difference in a quasistatic electric sheath of

$$
\Delta \Phi=\frac{E_{\mathrm{kin}}^{\max } \times A}{e Z}=\frac{E_{\mathrm{kin}}^{\max }}{e(q / m)}=(5.17 \pm 0.27) \mathrm{MV},
$$

where $E_{\text {kin }}^{\max }$ is given in units of $\mathrm{MeV} / \mathrm{u}$ and $A$ is the ion's mass number. It is the highest field potential experienced by ions in that shot exceeding also the field potential experienced by protons which is $\Delta \Phi=(4.6 \pm 0.2) \mathrm{MV}$. This indicates that the recorded protons have to originate from hydrogen stored in the bulk on interstitials of the atomic lattice and not from a hydrogen layer accumulated on the target rear surface. Earlier experiments on heavy ion induced desorption [30] have shown that hydrogen diffuses from the bulk to the surface and therefore could not be removed completely. Thus, the ion induced desorption is mainly a surface effect. Similar observations were made in the case of the fs-laser desorption. The adsorbed gases on the target surface could be removed quite well which is illustrated by the acceleration of aluminum ions of several charge states but also by a number of gold and copper ion traces in the respective shots. But neither the oxide layer on top of copper and aluminum foils as well as some carbon remnants on gold foils nor the hydrogen stored in the bulk of these materials could be removed completely and this led to the acceleration of oxygen and carbon ions as well as of protons.

In ns- to ps-laser ablation [22] carbon and oxygen ions with low charge states $\left(\mathrm{C}^{+}, \mathrm{C}^{2+}, \mathrm{O}^{+}, \mathrm{O}^{2+}\right)$ are still present which could be due to a still thick enough contamination layer or due to the longer laser pulse interacting with 
the ablated material. Cleaning techniques as resistive heating [13] dehydrogenize the target completely with the consequence that no protons were observed at all. In contrast, our data show that a stepwise increase of the intensity of the desorption laser provides a consecutive decrease of the contamination layer. Thus, this new cleaning technique allows one to scan through the contamination layer investigating different stages of the cleaning process. No other cleaning technique could provide this possibility, so far.

\section{CONCLUSION AND OUTLOOK}

The intention of our experiment was the investigation of the influence of fs-laser desorption on the proton and ion acceleration induced by the TNSA mechanism. Thin metal foils were substantially cleaned from hydrocarbon contaminations and oxygen layers by irradiating these foils with a variable amount of short laser pulses (90 fs) at $10 \mathrm{~Hz}$ repetition rate with intensities on the order of $10^{12}-10^{13} \mathrm{~W} / \mathrm{cm}^{2}$. Between the individual pulses and after the arrival of the last cleaning pulse residual gas atoms readsorbed on the target surface creating some few monolayers until the ion acceleration was induced by the high intensity CPA beam of $(3.1-6.9) \times 10^{19} \mathrm{~W} / \mathrm{cm}^{2}$. The simulated threshold intensities for melting of the target given in this paper matched well with our experimental findings for gold and copper targets. In case of aluminum, however, the experimentally achievable desorption intensities without causing observable damage to the target foil lay about 7 times above the calculated intensity thresholds. The underestimation of threshold intensities from theoretical models gets even higher when a reduced reflectance due to contaminants on the target surface is considered. This discrepancy is probably due to a lower intensity within the material than assumed, which may be connected with the larger amount of contaminations on the surface of aluminum compared to the other metals.

Within the experiment we have demonstrated a new method to strongly modify the ion energy spectrum of TNSA-ion beams. An increase of the intensity $I_{\text {des }} \geq$ $7.0 \times 10^{12} \mathrm{~W} / \mathrm{cm}^{2}$ and quantity of the desorption laser pulses provided a drop of the maximum proton energy by at least a factor of 2 and a drop of the proton intensity by one order of magnitude compared to the shots without applied laser desorption. A complete removal of protons was not possible. This had several reasons. For some shots, either the intensity of the desorption laser beam or the quantity of cleaning pulses did not suffice. For other shots, the residual gas pressure in the target chamber was high enough such that readsorption between the individual pulses became an issue. Assuming an extensive removal of hydrogen on the target rear surface, the observed proton signals have to originate from hydrogen stored in the bulk of the target. This supposition applies to the discussed shot on an aluminum foil in which the electric field potential experienced by $\mathrm{O}^{6+}$, superposed with $\mathrm{Al}^{10+}$, was $0.6 \mathrm{MV}$ higher than that experienced by protons. This means that these ions have to form the uppermost layer being exposed to the electric field.

Further common modifications of the TNSA-ion spectra result from the fs-laser desorption. These are the strong reduction or even the complete disappearance of ions with low charge states, an intensified signal of $\mathrm{C}^{4+}$ and $\mathrm{O}^{6+}$ in the higher energetic end of their trace, and the acceleration of metal ions from the target material $(\mathrm{Au}, \mathrm{Cu}, \mathrm{Al})$. The specific characteristics of the ion spectra depends on several parameters. These are the thickness as well as the composition of the contamination layer, the readsorption process, and the TNSA electric field strength. The electric field potential ionizes and accelerates surface atoms with highest available charge-to-mass ratio first and gets screened for atoms in deeper layers. Because of its energy transfer to atoms it diminishes rapidly in time as the laser pulse is too short to admit electron recirculation through the target foil. Since in most shots the electric field energy was not high enough to ionize atoms up to their $K$ shell and to accelerate them we observed enhanced signals of $\mathrm{C}^{4+}$ and $\mathrm{O}^{6+}$, i.e., both ions with completely ionized $L$ shell. The reduction of ions with low charge states is a direct consequence of a substantial decrease of the contamination layer thickness. When no more contaminants are present the remaining field energy is transferred to the ionization and acceleration of target ions. This is exactly what we observed in our data.

The fs-laser desorption has been proven to be an excellent method to influence TNSA ions and to learn more about the TNSA field potential by a successive removal of contaminants. The possibility of selecting a targeted range of ion species for the acceleration process in a determined energy window as a future perspective of this method would offer new scientific applications in many fields of research. It represents one important step towards monoenergetic ion beams driven by ultraintense laser pulses. With a further collimation of these beams by electromagnetic devices, they could compete with conventionally generated ion beams and all its applications, one day.

In order to extend the knowledge of fs-laser desorption followed by the TNSA further investigations of its individual physical processes, i.e., the fs-laser desorption itself, the readsorption process, the ionization and the subsequent ion acceleration, have to be made. A thorough analysis of the target composition with atomic depth profiles is of great importance, too. A significant milestone towards a controlled surface modification of a characterized target by fs-laser desorption represents the in situ measurement of the desorbed material directly after the irradiation. Apart from more experimental data at different laser parameters, theoretical studies on this complex topic are indispensable. A novel numerical modeling of field ionization in particlein-cell codes to analyze the cutoff energies of the laseraccelerated carbon ions was developed by the group of 
Nuter [31]. With this work numerical investigations of different carbon ion charge states are available while the usual simulation codes for TNSA consider only the accelerated protons. The first comparison of this model with our experimental results gives a qualitatively good agreement. Combining this code with simulations of the fs-laser desorption process might give a profound access to the theoretical understanding of the influence of fs-laser desorption on the ion acceleration.

\section{ACKNOWLEDGMENTS}

The authors would like to thank the Callisto laser crew of the Jupiter Laser Facility at Lawrence Livermore National Laboratory, California, for their excellent support at any time during our experimental campaign. The experiment was funded by Bundesministerium für Bildung und Forschung (BMBF), support code 06 DA 9044 I. We further thank the DFG for their contribution within the Emmy Noether Project No. RE 1141/11-1. The use of the Jupiter Laser Facility was supported by the U.S. Department of Energy, Lawrence Livermore National Laboratory, under Contract No. DE-AC52-07NA27344.

[1] S. P. Hatchett et al., Phys. Plasmas 7, 2076 (2000).

[2] R. A. Snavely, M. H. Key, S. P. Hatchett, T. E. Cowan, M. Roth, T. W. Phillips, M. A. Stoyer, E. A. Henry, T. C. Sangster, M.S. Singh, S.C. Wilks, A. MacKinnon, A. Offenberger, D. M. Pennington, K. Yasuike, A. B. Langdon, B. F. Lasinski, J. Johnson, M.D. Perry, and E. M. Campbell, Phys. Rev. Lett. 85, 2945 (2000).

[3] T. E. Cowan et al., Phys. Rev. Lett. 92, 204801 (2004).

[4] K. W. D. Ledingham, P. McKenna, and R. P. Singhal, Science 300, 1107 (2003).

[5] A. Mancic, J. Robiche, P. Antici, P. Audebert, C. Blancard, P. Combis, F. Dorchies, G. Faussurier, S. Fourmaux, M. Harmand, R. Kodama, L. Lancia, S. Mazevet, M. Nakatsutsumi, O. Peyrusse, V. Recoules, P. Renaudin, R. Shepherd, and J. Fuchs, High Energy Density Phys. 6, 21 (2010).

[6] A. Pelka et al., Phys. Rev. Lett. 105, 265701 (2010).

[7] C. K. Li, F. H. Séguin, J. A. Frenje, J. R. Rygg, R. D. Petrasso, R. P. J. Town, P. A. Amendt, S. P. Hatchett, O. L. Landen, A. J. Mackinnon, P. K. Patel, V.A. Smalyuk, T.C. Sangster, and J.P. Knauer, Phys. Rev. Lett. 97, 135003 (2006).

[8] M. Roth, T.E. Cowan, M. H. Key, S. P. Hatchett, C. Brown, W. Fountain, J. Johnson, D. M. Pennington, R. A. Snavely, S.C. Wilks, K. Yasuike, H. Ruhl, F. Pegoraro, S. V. Bulanov, E. M. Campbell, M. D. Perry, and H. Powell, Phys. Rev. Lett. 86, 436 (2001).

[9] M. Temporal, R. Ramis, J. J. Honrubia, and S. Atzeni, Plasma Phys. Controlled Fusion 51, 035010 (2009).

[10] S. V. Bulanov and V.S. Khoroshkov, Plasma Phys. Rep. 28, 453 (2002).
[11] S. D. Kraft et al., New J. Phys. 12, 085003 (2010).

[12] S. C. Wilks, A. B. Langdon, T.E. Cowan, M. Roth, M. Singh, S. Hatchett, M. H. Key, D. Pennington, A. MacKinnon, and R.A. Snavely, Phys. Plasmas 8, 542 (2001).

[13] B. M. Hegelich, B. J. Albright, J. Cobble, K. Flippo, S. Letzring, M. Paffett, H. Ruhl, J. Schreiber, R. K. Schulze, and J. C. Fernández, Nature (London) 439, 441 (2006).

[14] H. Schwoerer, S. Pfotenhauer, O. Jäckel, K.-U. Amthor, B. Liesfeld, W. Ziegler, R. Sauerbrey, K. W. D. Ledingham, and T. Esirkepov, Nature (London) 439, 445 (2006).

[15] S. Ter-Avetisyan, M. Schnürer, P. V. Nickles, M. Kalashnikov, E. Risse, T. Sokollik, W. Sandner, A. Andreev, and V. Tikhonchuk, Phys. Rev. Lett. 96, 145006 (2006).

[16] http://nobelprize.org/nobel_prizes/chemistry/laureates/2002/.

[17] C. Frischkorn and M. Wolf, Chem. Rev. 106, 4207 (2006).

[18] S. Wagner, H. Öström, A. Kaebe, M. Krenz, M. Wolf, A. Lunz, and C. Frischkorn, New J. Phys. 10, 125031 (2008).

[19] W. S. Fann, R. Storz, H. W. K. Tom, and J. Bokor, Phys. Rev. B 46, 13592 (1992).

[20] B. Rethfeld, A. Kaiser, M. Vicanek, and G. Simon, Phys. Rev. B 65, 214303 (2002).

[21] R.E. Russo, X. Mao, J. J. Gonzalez, and S. S. Mao, J. Anal. At. Spectrom. 17, 1072 (2002) [http://pubs.rsc.org/ en/Content/ArticleLanding/2002/JA/b202044k].

[22] J. C. Fernández, B. M. Hegelich, J.A. Cobble, K. A. Flippo, S. A. Letzring, R. P. Johnson, D. C. Gautier, T. Shimada, G. A. Kyrala, Y. Wang, C. J. Wetteland, and J. Schreiber, Laser Part. Beams 23, 267 (2005).

[23] B. L. Doyle and P. S. Peercy, Appl. Phys. Lett. 34, 811 (1979).

[24] D. S. Ivanov and L. V. Zhigilei, Phys. Rev. B 68, 064114 (2003).

[25] S.I. Anisimov, A.M. Bonch-Bruevich, M. A. El'yashevich, Y.A. Imas, N. A. Pavlenko, and G. S. Romanov, Sov. Phys. Tech. Phys. 11, 945 (1967).

[26] S. I. Anisimov, B. L. Kapeliovich, and T. L. Perel'man, Sov. Phys. JETP 39, 375 (1974).

[27] Here we assume that the target chamber pressure is homogeneous although the local pressure at the target surface might be much higher due to the desorption. This higher local pressure would lead to an increased readsorption rate.

[28] K. Harres, M. Schollmeier, E. Brambrink, P. Audebert, A. Blažević, K. Flippo, D. C. Gautier, M. Geißel, B. M. Hegelich, F. Nürnberg, J. Schreiber, H. Wahl, and M. Roth, Rev. Sci. Instrum. 79, 093306 (2008).

[29] F. Nürnberg, M. Schollmeier, E. Brambrink, A. Blažević, D. C. Carroll, K. Flippo, D. C. Gautier, M. Geißel, K. Harres, B. M. Hegelich, O. Lundh, K. Markey, P. McKenna, D. Neely, J. Schreiber, and M. Roth, Rev. Sci. Instrum. 80, 033301 (2009).

[30] M. Bender, H. Kollmus, H. Reich-Sprenger, M. Toulemonde, and W. Assmann, Nucl. Instrum. Methods Phys. Res., Sect. B 267, 885 (2009).

[31] R. Nuter, L. Gremillet, E. Lefebvre, A. Lévy, T. Ceccotti, and P. Martin, Phys. Plasmas 18, 033107 (2011). 